\title{
Design of Small Intelligent Adaptation Auxiliary Equipment Based on Communication Antenna
}

\author{
Shuhua Yin \\ Xi'an Fanyi University, Xi'an 710065, China \\ Shuhua_y@126.com
}

\begin{abstract}
A small intelligent adaptive auxiliary device which can automatically detect the multi-antenna transmission efficiency and recognize the strength of received signal and automatically switch the antenna is designed, aiming at the shortcomings of the shortcomings of the shortwave wireless communication equipment of national defense communication network. Single-chip is used for full digital control, adaptive digital filtering and fuzzy processing and Chinesization display and other key technologies to complete the communication antenna detection and automatic switching and alarm functions to achieve the best receiving results of short-wave wireless communications equipment antenna. After six months of trial run through networking, the probability of abnormal running has not yet found.
\end{abstract}

Keywords: Short-Wave communication; Intellegent device; Auxiliary equipment; Fuzzy processing; Power measurement

\section{基于通信天线的小型智能适配辅助设备的设计}

\author{
尹树华 \\ 西安翻译学院, 陕西省 西安 710065
}

摘要: 针对国防通信组网的短波无线通信设备的天线在使用中存在的缺陷问题, 设计一种能够自动检测多副天线发射效 率和识别接收信号强弱能力以及自动切换天线的小型智能适配辅助设备。采用单片机进行全数字化控制、自适应数字滤波和 模糊处理及汉化显示等关键技术, 完成通信天线的检测与自动切换及报警等功能, 达到短波无线通信设备天线接收最佳效果。 通过组网进行半年的试运行, 尚未发现运行不正常概率。

关键词: 短波通信; 智能适配; 辅助设备; 模糊处理; 功率测量

中图分类号：TN 82 文献标志码：A

引言

短波无线通信设备是国防战略通信组网的核心要素, 国防通信任务的特殊性决定了短波无线通信设备 重要性, 在特定的条件下执行特殊应急任务, 通常使用短波无线通信设备实施通信保障。短波无线通信设 备进行信息沟通依靠高质量的天线, 现有短波通信设备通常使用宽带、三环、双极三种分立天线, 在开通 运行、值勤操作、使用维护时往往存在缺陷: 使用前不知道天线的好坏; 通信过程中要有人随时看护; 无 法对天线的好坏进行评估; 一旦通信设备天线损坏, 通信会长时间阻断。为此需要设计一种能够自动检测 多副天线发射效率和识别接收信号强弱能力以及自动切换通信设备天线的智能适配辅助设备。天线智能适 配辅助设备利用单片机、全数字化控制、汉化显示等技术 ${ }^{[1][2]}$, 实现了通信设备三种天线的检测与自动切 换，对损坏的天线报警并自动切换到正常的天线上，最大程度地发挥短波无线通信设备的效能 ${ }^{[3][4]}$ 。

\section{1 总体方框构成}

小型智能适配辅助设备由模拟信号处理模块, 射频频率检测模块、射频功率检测模块, 模拟信号处理 
模块, 数字控制模块和现代的数字信号处理模块以及其他辅助模块等构成。方框图如图 1 所示。

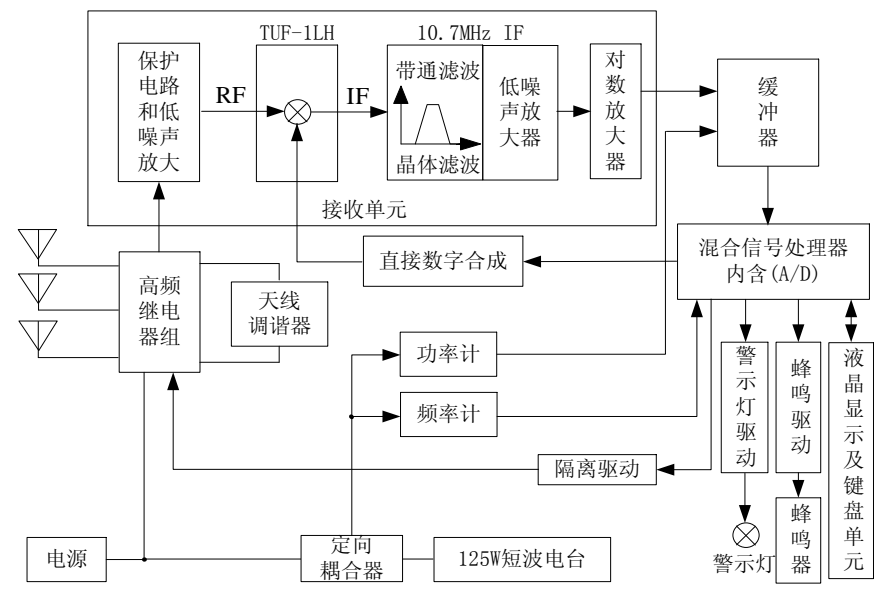

图 1 小型智能适配辅助设备方框图

\section{2 辅助设备电路设计}

小型智能适配辅助设备在短波无线通信设备内的天调位置与天线间加入一个切换辅助设备, 通过检测 信号的强弱来命令单片机去控制切换辅助设备, 完成对天线的选择性使用。电路设计如图 2 所示 ${ }^{[5]}$ 。

\section{1 辅助设备辅助通道}

工作过程是辅助通道是通过天线耦合一定量的信号，经过宽带低噪声放大器进行放大后，再通过一个 自动增益控制的增益级, 最终由超高速模数转换器变为数字信号, 通过数字信号处理器进行处理, 以实现 选频、变频、强度检测、解调。数字信号处理器主要基于软件工作, 而相对硬件要求较少。天线信号是通 过定向耦合器耦合, 依据要求确定耦合度。若只是用于作信号强度估计, 则可只耦合 $-20 \mathrm{~dB}$; 若要对信号 进行解调, 则要求耦合 $-3 \mathrm{~dB}$, 这时辅助设备的辅助通道将成为一个辅助接收机 ${ }^{[6]}$ 。

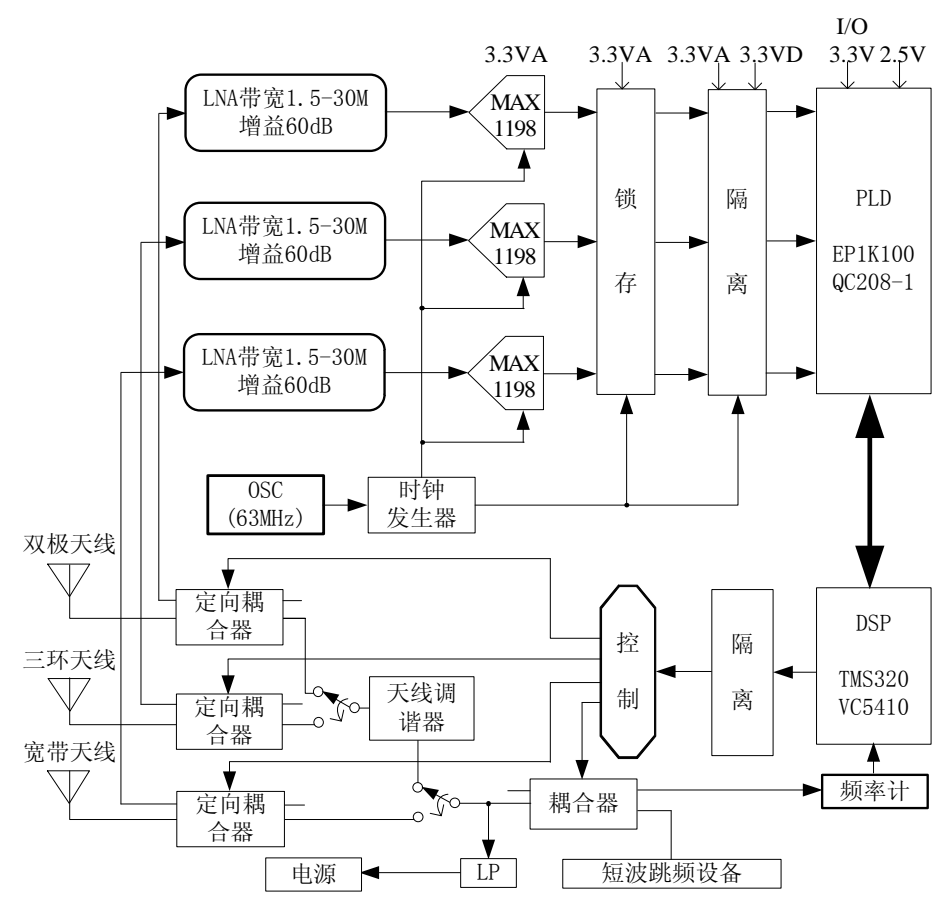

图 2 小型智能适配辅助设备电路设计图 


\section{2 单片机与切换}

在单片机控制下接收键盘送来的命令, 在单片机控制下转换成对切换的控制信号。为了避免数字部件 对电气前端信号的干扰和影响, 将控制信号要进行隔离。切换设备是完成各天线, 天调, 通信设备之间的 连接, 单片机送来控制信号后, 完成相应的切换动作。这部分采用大功率电子开关或继电器实现, 不管哪 种元器件, 都要考虑插入损耗, 驻波比, 阻抗匹配和隔离度等问题。

\section{3 设计的基本方案}

小型智能适配辅助设备设计的难点: 频率功率测量; 强电磁场干扰影响弱信号电路的正常工作; 自适 应数字滤波和模糊处理。频率功率测量是实现自适应运算的主要参数来源和控制依据, 后两项则是设备赖 以正常运行的软硬件基础和基本条件，因此技术实施及试验调试过程有难度，基本方案的设计慎之又慎。

\section{1 功率与频率的测量技术}

\subsection{1 功率的测量 ${ }^{[7][8]}$}

在发射部分，通信设备正常工作时发射功率可达 130W，强射频信号混合在系统中势必造成设备无法正 常工作。在设计时将发射功率的采样和其它电路完全独立分开。高频互感器适当的定向耦合器的特性。为 此设计了 “ $\Gamma$ 形” 发射功率及反射功率测量的取样电路完成。

通过计算在主线路上阻抗匹配时, VPFW 端电压值完全正比于正向传输（发射）功率, VPBW 端电压值 完全正比于反向传输 (反射) 功率; 阻抗失配时, VPFW 端电压值代表正向传输 (发射) 功率的变化趋势, $\mathrm{VPBW}$ 端电压值代表反向传输 (反射) 功率变化趋势。选择合适参数, 精心调整电路, 使插入损耗小于 $0.1 \mathrm{~dB}$, 驻波比没有明显增加。

在接收部分, 采用 AGC 技术, 不难获得较好的短波接收性能, 产生的负面影响是 AGC 技术掩盖了真实 的接收信号电平。如果舍弃 AGC 技术, 势必造成接收机动态范围的降低。增益设计低, 对强信号有利, 但 灵敏度明显降低; 增益设计高, 对弱信号有利, 但又容易产生自激并且强信号会被限幅而不能正常检测信 号电平, 造成 “阻塞”, 使接收机不能工作。

当发射机接通一副天线时，接收机接通其它的天线，保证强发射功率能量不会直接进入接收机。一个 快速的检测电路检测接收通道, 并且当检测到有强信号电平存在时立刻切断接收输入网络, 同时在接收机 前端设计一个大功率高速限幅器, 使接收机的保险性能大大提高。对数接收机的输出已解调成基带信号。 对于常规报务、话务调幅应用, 已可完全表达接收功率的大小。对于单边带信号, 经过一定的换算也可代 表接收功率的强弱。

\subsection{2 频率的测量}

DDS 作为数字本振是测频的主要目的, 由于收发同频, 可测量发射信号频率来代表通信设备工作频率。 把发射机功率测量采样样本分出极小部分, 经高频频率计测出当前发射信号频率或周期。所测量的是调制 后信号, 而不是载波, 此时该信号的瞬时频率或周期与载波有一定的误差, 不能认为是通信设备工作频率。 利用信号的随机性, 调制载波后所形成的频偏同样具有随机性, 通过多次测量求取期望值, 即认为是通信 设备工作频率的估值。

\section{2 自适应滤波和模糊处理技术}

发射天线是否可用的重要指标由驻波比衡量, 由于通信设备使用了天线调谐系统, 有时在不接天线的 
情况下或天线严重损坏, 天线调谐也能进行不必要的补偿, 往往掩盖了发射系统严重失配的参数差异, 这 对判别发射天线性能优劣造成了极大的困难。随着工作环境温度的改变, 器件的工作参数会有差异。解决 办法是将各项参数全部数字化, 采用数字的方法加以处理。实施步骤为: 确定系统无任何信号时的本底噪 声 N0; 确定系统连接天线而无接收信号时的基本噪声 N1; 测量发射信号时的发射功率和反射功率; 测量 接收信号时的信号功率; 将所有数据进行滤波平滑，剔除离散极大的数据; 计算信噪比和驻波比。采用模 糊推理原则，将天线收发性能综合指标排序，即得天线自适应控制的有效依据。

\section{4 结语}

小型智能适配辅助设备完成的主要功能是：操作和界面人性化, 液晶屏进行人机对话, 显示天线工作 状态、通信工作频率、当前信号的强弱等。所有操作都使用简单的几个按键来实现, 高效方便。适配装置 的操作分手动和自动。自动功能: 若适配装置设置在自动功能状态下, 将检测当前天线的发射功率和接收 信号的强弱; 若通信设备在发射功率未达到发射指标, 将通过蜂鸣器和液晶屏幕进行提示, 同时自动切换 到另一种天线进行发射; 若天线正常, 在发射完毕后, 将自动检测三种天线接收信号的强弱, 并选择信号 最强的天线作为接收天线; 在自动状态下, 自动检测当前的发射频率, 并自动修改工作频率，达到最好的 接收效果。

\section{4 致谢}

本研究是陕西省教育厅专项科研计划项目, 项目编号为 16JK2078，由陕西省教育厅自然科学研究基金 资助, 对此表示衷心感谢。

\section{Acknowledgement}

In this paper, the research was sponsored by the nature science research projects of education department in Shaanxi Province government and key project of education department in Shaanxi provincial government (Project No. 16JK2078).

\section{参考文献:}

[1] 刘振岭, 张水兴, 李骏. 短波通信网在应急通信领域的应用 $[\mathrm{J}]$ ．无线电通信技术．2014(02)：82-85.

[2] 姬五胜, 张丰臣, 刘培涛. 移动通信基站天线的关键技术研究进展 [J]。电讯技术. 2017 (01)：111-117.

[3] 胡泓涛. 某型短波自适应通信系统网络信号实时分析及入侵监测研究 [D]. 电子科技大学 2015 .

[4] 杨华, 兰明蛟, 王星全.一种机动架设高增益短波天线设计 [J]。现代防御技术．2014(04)：103-107.

[5] 李引凡, 陈政, 邱洪云. 天线调谐器 $\mathrm{T}$ 形阻抗匹配网络参数估算 $[\mathrm{J}]$ ．现代电子技术．2013(08)：7-12.

[6] 张红梅, 韩万刚.一种新的变步长 LMS 自适应滤波算法研究及其应用 [J]．仪器仪表学报．2015 (08)：1822-1830.

[7] 钱其, 康宇, 赵云波. 基于改进的变步长自适应滤波算法的功率测量 $[J]$. 中国科学技术大学学报. 2015, 45(10): 855-863.

[8］张先庭, 陈琼, 邓洪峰．交流功率测量相位偏移补偿的研究 [J]．电测与仪表．2013(01)：42-46.

\section{References:}

[1] Liu Zhenling, Zhang Shuixing, Li Jun. Application of Shortwave Communication Network in Emergency Communication Field [J]. Radio Communication Technology. 2014 (02): 82-85.

[2] Ji Meisheng, Zhang Fengchen, Liu Peitao. Progress of Study on the Key Technology of Mobile Communication Base Station Antenna [J]. Telecommunications Technology. 2017 (01): 111-117. 
[3] Hu Hongtao. Real-time Analysis of A Short-Wave Adaptive Communication System Network Signal and Intrusion Detection Study [D]. Electronic Science and Technology University 2015.

[4] Yang Hua, Lan Mingjiao, Wang Xingquan. Design of a Mobile Erected High-Gain Short-Wave Antenna [J]. Modern Defense Technology. 2014 (04): 103-107.

[5] Li Zhifen, Chen Zheng, Qiu Hongyun. Antenna Tuner T-shaped Impedance Matching Network Parameter Estimation [J]. Modern Electronics Technique. 2013 (08): 7-12.

[6] Zhang Hongmei, Han Wangang. The Study of a New Variable Step Size LMS Adaptive Filtering Algorithm and Its Application [J]. Instrument and Meter Academic Journal. 2015 (08): 1822-1830.

[7] Qian QI, Kang Yu, Zhao Yunbo. Power Measurement Based on Improved Variable Step Size Adaptive Filtering Algorithm [J]. Journal of University of Science and Technology of China, 2015, 45 (10): 855-863.

[8] Zhang Xianting, Chen Qiong, Deng Hongfeng. Study on AC Power Measurement Phase Offset Compensation [J]. Electrical Measurement and Instrumentation. 2013 (01): 42-46. 